\title{
Pseudogout: Uncommon and Under-Recognized
}

\author{
Scott R. Brown, DO'; Alissa M. Cohen, MS, DO'1 \\ 'University of Pittsburgh Medical Center, Shadyside Hospital, Pittsburgh, PA
}

\section{KEYWORDS:}

Chondrocalcinosis

CPPD

CPPD Disease

Calcium Pyrophosphate

Deposition Disease

Pseudogout
ABSTRACT: Pseudogout or calcium pyrophosphate deposition (CPPD) disease is an uncommon and often under-recognized presentation in primary care. Patients may initially develop asymptomatic crystal deposition, but these changes can evolve to synovitis, arthritis, and cartilage calcification. An initial differential diagnosis includes traditional gout, osteoarthritis, rheumatoid arthritis, septic arthritis, and Lyme disease. Acute attacks of CPPD may be indistinguishable from these conditions clinically, and a definitive diagnosis requires synovial fluid analysis. Fluid should be microscopically analyzed for cell count, crystal analysis under compensated polarizing microscopy, Gram stain, and culture. CPPD crystals are weakly birefringent under polarized light and have a rhomboid or rod-shaped appearance. No therapy is proven optimal, so CPPD treatment is instead tailored to symptoms, with goals of controlling acute pain, preventing additional attacks, and impeding the degenerative joint disease associated with CPPD disease arthropathy. Current treatment methods include intra-articular corticosteroid injections, NSAIDs, DMARDs, OMT, and in severe, refractory cases, surgery.

\section{INTRODUCTION/EPIDEMIOLOGY}

Calcium Pyrophosphate Deposition disease, also known as CPPD disease or Pseudogout, is an uncommon and often under-recognized presentation in primary care. While the exact prevalence of this condition is unknown, it is estimated that $4-7 \%$ of adults in the U.S. are affected. ${ }^{1-3}$ CPPD disease is generally seen in patients over age 60 , with risk increasing with age, while a majority of cases are found in the setting of prior arthritis., ${ }^{2,3}$ This review summarizes the evaluation and management of this polymorphous cause of crystal-induced arthritis.

\section{DIFFERENTIAL}

CPPD disease is likely under-recognized as a cause of both acute and chronic synovitis and arthritis given its wide range of clinical presentations, as well as the much more common conditions that mimic its presentation. ${ }^{1}$ While patients may initially develop

\section{CORRESPONDENCE:}

Scott R. Brown, DO | brownsr4@upmc.edu asymptomatic crystal deposition, these changes can evolve to synovitis and arthritis, and even cartilage calcification. Given these features, an initial differential diagnosis includes traditional gout, osteoarthritis, rheumatoid arthritis, septic arthritis, and Lyme disease, among other causes of monoarticular arthropathy.

\section{HISTORY}

Patients with CPPD disease are generally older than age 60, with prevalence doubling each decade after that. ${ }^{2,3}$ To further complicate the initial assessment, those with CPPD generally have a history of prior joint damage or degeneration, such as gout, osteoarthritis, or trauma. ${ }^{1-3}$ CPPD disease can affect any joint, but most commonly affects the knees, wrists, hips, symphysis pubis, and metacarpophalangeal joints. ${ }^{1}$

Patients have joint pain with joint tenderness and swelling. Patients may endorse symptoms of systemic illness including fevers, chills, and malaise. A prior family history of CPPD disease may help make the diagnosis. Several medications and metabolic conditions are associated with CPPD disease attacks, including loop diuretics, pamidronate, and intra-articular hyaluronic acid injections, as well as hyperparathyroidism, hypomagnesemia, and hemochromatosis. ${ }^{1-3}$ 


\section{TABLE 1:}

Comparison of pseudogout and gout

\begin{tabular}{|c|c|c|}
\hline & Pseudogout & Gout \\
\hline EPIDEMIOLOGY & Age >60; affects males and females equally & Age 30-60; affects males more commonly than females \\
\hline ETIOLOGY & Chondrocalcinosis & Hyperuricemia \\
\hline JOINT PREDILECTION & Larger joints, most commonly the knee & Smaller joints, most commonly the first metatarsophalangeal joint \\
\hline CRYSTAL APPEARANCE & Weakly birefringent, rhomboid- or rod-shaped & Negative birefringence, needle- shaped \\
\hline
\end{tabular}

\section{CLINICAL EVALUATION}

CPPD disease is defined by acute attacks of joint pain and swelling due to synovitis that mimic gout (Table 1). These acute or subacute attacks can involve one or multiple joints. Similar to gout, CPPD disease can manifest with elevated acute-phase reactants, including ESR and CRP levels. Acute attacks of CPPD may be indistinguishable from acute gout. ${ }^{4}$ One cannot definitively diagnose either condition without a synovial fluid analysis. Although CPPD disease and gout share similar joint predilection, CPPD disease tends to affect larger joints more commonly than gout and smaller joints less commonly than gout.,

\section{DIAGNOSTICS}

Because acute CPPD disease closely resembles gout, the definitive diagnosis requires synovial fluid analysis. ${ }^{4}$ Synovial fluid should be microscopically analyzed for cell count and crystal analysis under compensated polarizing microscopy. In addition, fluid should be examined by Gram stain and culture. CPPD crystals are weakly birefringent under polarized light and have a rhomboid or rodshaped appearance, while gout crystals are needle-shaped with negative birefringence. ${ }^{4,5}$ Crystals can be seen either intracellularly or extracellularly; however, detection might not be as accurate if fluid analysis is delayed. ${ }^{4}$ CPPD disease and gout can also coexist. ${ }^{4}$

Radiographs can show chondrocalcinosis in the involved joint and other joints even if CPPD disease is not clinically active at the time of presentation. Radiographs can help confirm the clinical impression, especially images of the knees, wrists, and anterior pelvis, as well as determining the extent of joint degeneration; however, radiographs are not required to make the diagnosis once CPPD crystals are seen under polarized light., ${ }^{6,7}$ Chondrocalcinosis is seen in the knees, wrists, and other joints such as intervertebral discs and the symphysis pubis., ${ }^{4,7}$ Other radiographic features include joint space narrowing, subchondral bone formation, normal bone mineralization, cysts more prominent than in osteoarthritis, bilateral preponderance, and osteophyte formation. ${ }^{6}$

Most of the differential diagnosis factors with gout can be considered in the case of CPPD and must be ruled out. Infection is always a major differential, especially in the patient presenting with acute monoarticular arthritis. In addition, septic arthritis can coexist in a joint that has been, or is currently, involved in an acute CPPD disease attack, as with gout. ${ }^{2-4}$ Thus, it is important to aspirate the involved joint whenever possible for the microscopic examination of the synovial fluid and Gram stain and culture.

\section{TREATMENT}

Optimal therapy includes prompt treatment of the acute attack, prevention of additional attacks, and prevention or reversal of the degenerative joint disease associated with CPPD disease arthropathy. Unfortunately, no proven therapy fits this description for CPPD, and no treatment is available to dissolve the crystal deposits. ${ }^{4,5}$

The treatment of CPPD disease is mostly tailored to the manifesting symptoms. In patients presenting with one or two joints of acute synovitis, after septic arthritis has been ruled out, rapid relief of pain and inflammation may be accomplished with joint aspiration and steroid injection. ${ }^{4,6}$ Many patients find relief from the joint aspiration itself. When more than two joints are involved, it is not feasible to inject all the joints, so treatment is directed toward systemic therapy, with nonsteroidal anti-inflammatory drugs, like indomethacin and naproxen. For patients unable to tolerate these agents, colchicine is another alternative, but it typically needs to be given three to four times daily to be effective.

Other medicines may help some patients during severe attacks of CPPD disease or with the less common chronic inflammation that these crystals can cause. These drugs include hydroxychloroquine, methotrexate, or an interleukin-1 beta antagonist medication which can decrease inflammation., 3,4,6,8 The successful use of anakinra, an interleukin-1 receptor antagonist, has been described for treatment and prophylaxis of acute CPPD arthritis resistant to NSAIDs and prednisolone. ${ }^{8}$

All patients should receive education about their disease, a prescription for physical therapy for local strengthening and aerobic exercise, advice on reduction of adverse mechanical factors, and simple analgesia. Goals of treatment include control of symptoms, early mobilization to avert effects of prolonged immobility, and maintenance or improvement of function. ${ }^{3,4}$ 
Osteopathic Manipulative Treatment (OMT) is another option to address musculoskeletal complaints. There are many known benefits to OMT including increased range of motion, decreased pain, improved ADL's, and shortened disability time. ${ }^{9}$ While there are no direct studies on treating CPPD with OMT, after obtaining a history and choosing an appropriate technique, OMT can be considered as another treatment option.

In severe cases of CPPD, surgery to repair and replace damaged joints may be required. CPPD management remains eminencebased, rather than evidence-based, as very few controlled clinical trials have been published. . $^{3,4} 6$

\section{AUTHOR DISCLOSURES:}

No relevant financial affiliations or conflicts of interest.

\section{REFERENCES:}

1. Abhishek A. "Calcium pyrophosphate deposition disease: a review of epidemiologic findings." Curr Opin Rheumatol. 2016 Mar;28(2):133-9. https://doi.org/10.1097/BOR.0000000000000246.

2. Richette P, Bardin T, Doherty M. "An update on the epidemiology of calcium pyrophosphate dihydrate crystal deposition disease." Rheumatology (Oxford). 2009 Jul;48(7):711-5. https://doi.org/10.1093/ rheumatology/kep081.

3. Rosenthal A, Ryan L. "Calcium pyrophosphate deposition disease." N Engl J Med. 2016 Jun 30;374(26):2575-84. https://doi.org/10.1056/ NEJMra1511117.

4. Guerne P, Terkeltaub R, Terkeltaub, R. "Calcium pyrophosphate dihydrate crystal deposition: epidemiology, clinical features, diagnosis, and treatment." gout and other crystal arthropathies. Elsevier Saunders. 2012. pp. 249-265.5.

5. Doherty M, Abhishek A, Hochberg M, Silman A, Smolen J. "Calcium pyrophosphate crystal-associated arthropathy". Rheumatology. Mosby Elsevier. 2011. pp. 1875-1887.

6. Zhang W, Doherty M, Pascual E. "EULAR recommendations for calcium pyrophosphate deposition. Part II: management". Ann Rheum Dis. vol. 70. 2011 Apr. pp. 571-5. https://doi.org/10.1136/ard.2010.139360.

7. Choi MH, MacKenzie JD, Dalinka MK. Imaging features of crystal-induced arthropathy. Rheum Dis Clin North Am 2006; 32:427-446. https://doi. org/10.1016/j.rdc.2006.04.001.

8. McGonagle D, Tan AL, Madden J, Emery P, McDermott MF. Successful treatment of resistant pseudogout with anakinra. Arthritis Rheum. 2008;58:631-3. https://doi.org/10.1002/art.23119.

9. Heinking K, Lipton J, Valashinas B. "Multiple small joint diseases in the elderly." Foundations of Osteopathic Medicine. Lippincott, Williams, \& Wilkins. 2011. pp. 958-9. 